\title{
AMCoR
}

Asahikawa Medical University Repository http://amcor.asahikawa-med.ac.jp/

Lower urinary tract symptoms (2015.1) 7(1):37-41.

Improvement of Overactive Bladder Symptoms and Bladder Ischemia with Dutasteride in Patients with Benign Prostatic Enlargement.

Wada Naoki, Matsumoto Seiji, Kita Masafumi, Hashizume Kazumi, Kakizaki Hidehiro. 


\title{
Title page
}

\section{Improvement of Overactive Bladder Symptoms and Bladder Ischemia with Dutasteride in Patients with Benign Prostatic Enlargement}

\author{
Naoki Wada, Seiji Matsumoto, Masafumi Kita, Kazumi Hashizume, Hidehiro Kakizaki \\ Department of Renal and Urologic Surgery, Asahikawa Medical University
}

Corresponding to: Naoki Wada, M.D, Ph.D

Department of Renal and Urologic Surgery, Asahikawa Medical University

2-1-1-1 Midorigaoka Higashi, Asahikawa, 078-8510, Japan

E-mail : nwada@asahikawa-med.ac.jp

TEL : +81 166682533

FAX : +81 166682539

Running title: dutasteride and bladder ischemia 


\section{ABSTRACT AND KEY WORDS}

\section{Objectives}

To evaluate the correlation of clinical and urodynamic parameters with bladder vascular resistance before and after dutasteride treatment in patients with lower urinary tract symptoms associated with benign prostatic enlargement.

\section{$\underline{\text { Methods }}$}

A prospective study was conducted in consecutive 30 patients with benign prostatic enlargement who had not been satisfied with alpha-adrenergic antagonist monotherapy. Before and 24 weeks after dutasteride add-on treatment, we assessed IPSS, prostate volume (PV), urodynamic study and contrast-enhanced color Doppler ultrasonography to measure bladder vascular resistive index (RI).

\section{$\underline{\text { Results }}$}

Twenty-four weeks after dutasteride, PV significantly decreased from $68 \pm 29$ to $48 \pm 28 \mathrm{ml}$ $(\mathrm{p}<0.001$ ), and there was significant improvement of IPSS (from 18.8 \pm 7.7 to $13.4 \pm 7.2, p<0.001$ ). Urgency score of IPSS was also significantly improved from $2.3 \pm 1.9$ to $1.4 \pm 1.4(p<0.01)$ after dutasteride. On pressure-flow study, bladder outlet obstruction index (BOOI) (from $58 \pm 36$ to 38 $\pm 27, \mathrm{p}<0.001$ ) and detrusor pressure at Qmax (PdetQmax) (from $73 \pm 34$ to $54 \pm 25 \mathrm{cmH}_{2} \mathrm{O}$, $\mathrm{p}<0.001$ ) were significantly improved. RI significantly decreased after dutasteride (from $0.548 \pm 0.069$ to $0.486 \pm 0.064, \mathrm{p}<0.001$ ). In 20 patients with persistent urgency after dutasteride, RI was less improved than in other 10 patients without urgency (change of RI $0.045 \pm 0.091$ vs. 0.096 $\pm 0.042, \mathrm{p}<0.05$ ). Posttreatment BOOI and PdetQmax in patients with persistent urgency was significantly higher than in those without urgency after dutasteride (BOOI: $46 \pm 28$ vs. $24 \pm 20$, p<0.05, PdetQmax: $62 \pm 26$ vs. $\left.40 \pm 17 \mathrm{cmH}_{2} \mathrm{O}, \mathrm{p}<0.01\right)$. 


\section{Conclusions}

Reduction of obstruction and improvement of bladder ischemia might play an important role in a beneficial impact of dutasteride on overactive bladder symptoms.

Key words: benign prostatic hyperplasia, bladder ischemia, dutasteride, lower urinary tract symptoms, overactive bladder 


\section{INTRODUCTION}

Benign prostatic obstruction (BPO) is diagnosed when the cause of bladder outlet obstruction (BOO) is known to be benign prostatic enlargement (BPE), due to histologic benign prostatic hyperplasia (BPH). In clinical practice, lower urinary tract symptoms (LUTS) suggestive of BPO (LUTS/BPO) in male patients are treated by pharmacological agents including $\alpha$-adrenergic antagonist and $5 \alpha$-reductase inhibitor. Dutasteride, a dual $5 \alpha$-reductase inhibitor, is used for treatment of LUTS associated with BPE. Previous studies showed that dutasteride with or without tamsulosin significantly improved not only voiding symptoms but also storage symptoms, ${ }^{1,2}$ including urgency that is the key symptom of overactive bladder (OAB) defined by The International Continence Society (ICS). ${ }^{3}$ However, there have been few reports that focused on the improvement of $\mathrm{OAB}$ by dutasteride and its mechanisms.

Experimental and clinical studies indicate that bladder ischemia is one of the important pathophysiological factors of OAB. We previously examined bladder vascular resistance by using color Doppler ultrasonography (CDUS) in patients with LUTS/BPO before and after TURP. ${ }^{4}$ In that study, we showed that higher bladder vascular resistance (bladder ischemia) before TURP was associated with higher degree of BOO and TURP decreased bladder vascular resistance. We also showed that less improvement of bladder vascular resistance after TURP was associated with postoperative persistent $\mathrm{OAB}$, implying that improvement of bladder ischemia is one of the critical factors for the improvement of $\mathrm{OAB}$ symptoms. Because dutasteride can reduce prostate size and relieve obstruction in patients with $\mathrm{BPE}{ }^{5}$ we hypothesized that dutasteride improves bladder ischemia and OAB symptoms in patients with BPE. In this prospective study, we evaluated the correlation of clinical and urodynamic 
parameters with bladder vascular resistance before and after dutasteride treatment in patients with LUTS associated with BPE.

\section{METHODS}

This study was approved by Asahikawa Medical University Ethical Committee and carried out in accordance with the principles of the Declaration of Helsinki. Each patient was informed of the nature and purpose of the study and informed consent was obtained.

A prospective study was conducted in consecutive 30 patients with LUTS associated with BPE who had not been satisfied with $\alpha$-adrenergic antagonist monotherapy. Inclusion criteria were prostatic volume (PV) $\geq 30 \mathrm{ml}$ and IPSS $\geq 8$ or QOL index $\geq 3$ under administration of any alpha-adrenergic antagonist (silodosin in 21 patients, tamsulosin in 6, and naftopidil in 3) without anticholinergic agent. Before and 24 weeks after dutasteride (0.5 mg daily) add-on treatment with preceding alpha-adrenergic antagonist, we assessed IPSS, PV, uroflowmetry (UFM), filling cystometry, pressure-flow study (PFS) and CDUS. The dose of alpha-adrenergic antagonist was not changed during the 24-week study period.

Urodynamic assessment included filling cystometry and PFS. Filling cystometry was performed with normal saline solution at a filling rate of $50 \mathrm{ml} / \mathrm{min}$. A $6 \mathrm{Fr}$ double-lumen transurethral catheter was used for infusion and recording intravesical pressure, and a hand-made intrarectal balloon catheter was used for recording abdominal pressure. The severity of obstruction was assessed using the bladder outlet obstruction index (BOOI) formerly known as the Abrams-Griffiths number, defined as detrusor pressure at maximum flow rate (PdetQmax) - 2Qmax. ${ }^{6}$ Detrusor overactivity (DO) was defined as involuntary detrusor contraction during filling phase irrespective of its amplitude. Otherwise the methods, definition, 
and units for filling cystometry conformed to the standards proposed by the ICS. ${ }^{3}$

Bladder vascular resistive index (RI) was measured by enhanced CDUS with ultrasound contrast agent (Levovist ${ }^{\circledR}$, Bayer, Tokyo, Japan), as previously reported (Figure 1). ${ }^{4}$ CDUS was performed by one examiner (NW) with ProSound SSD-5500 (ALOKA, Tokyo, Japan) equipped with a 5.0-MHz probe. CDUS was performed during the storage phase without urge to void with bladder volume between 100 and $150 \mathrm{ml}$. After the administration of contrast agent, arterial blood flow of lateral bladder wall was examined by a transabdominal ultrasonography. RI (Vmax-Vmin / Vmax) was measured and the average RI of right and left vessels was calculated.

To evaluate the correlation of clinical and urodynamic parameters with bladder vascular resistance before and after dutasteride treatment, we used Spearman's correlation for correlation analysis. To identify differences between before and 24 weeks after dutasteride treatment and between the patients with and without persistent urgency after dutasteride, a paired t-test and Student's t test was used, respectively. Persistent urgency was defined as $\geq 1$ point of question 4 of the IPSS after dutasteride treatment. $P<0.05$ was considered statistically significant.

\section{RESULTS}

The characteristics of the 30 patients who were treated with dutasteride add-on are shown in Table1. The mean age and PV of the study patients were 73.6 years (range 60-83) and $68 \mathrm{ml}$ (30-139), respectively. On filling cystometry, DO was shown in 23 patients (77\%).

Pretreatment RI did not statistically correlate with age ( $r=-0.14)$, IPSS $(r=0.08)$, maximum flow rate (Qmax) (r=0.18), PV ( $r=0.22, P>0.05)$, BOOI $(r=-0.04)$ or PdetQmax $(r=-0.02)$. 
There was no difference in pretreatment RI among three alpha-adrenergic antagonists. When the study patients were dichotomized using several cut-off values of these parameters, RI was significantly higher in patients with PV $\geq 60 \mathrm{ml}(n=18)$ than in those with $\mathrm{PV}<60 \mathrm{ml}(n=12)$ $(0.570 \pm 0.049$ versus $0.514 \pm 0.083, p<0.05)$. There was no significant difference in pretreatment RI among patients with different numbers of vascular risk factors or between patients with and without DO.

Twenty-four weeks after treatment with dutasteride, IPSS (from $18.8 \pm 7.7$ to $13.4 \pm 7.2, p<$ 0.001 ) and QoL index (from $4.6 \pm 1.0$ to $3.4 \pm 1.3$, $p<0.001$ ) were significantly improved (Table 2). Sub-scores of IPSS except Q6 (straining) were also significantly improved by dutasteride. $\underline{\text { Serum PSA level significantly decreased and serum testosterone level significantly }}$ increased after dutasteride. Qmax (from $12.2 \pm 6.1 \mathrm{ml} / \mathrm{sec}$ to $13.3 \pm 7.7 \mathrm{ml} / \mathrm{sec}, p=0.09$ ) and postvoid residual (from $93 \pm 88 \mathrm{ml}$ to $127 \pm 121 \mathrm{ml}, p=0.17$ ) did not change significantly. On PFS, BOOI (from $58 \pm 36$ to $38 \pm 27, p<0.001$ ) and PdetQmax (from $73 \pm 34 \mathrm{cmH}_{2} \mathrm{O}$ to $54 \pm$ $25 \mathrm{cmH}_{2} \mathrm{O}, p<0.001$ ) were significantly improved. Overall RI significantly decreased after treatment with dutasteride (from $0.548 \pm 0.069$ to $0.486 \pm 0.064, p<0.001$ ) (Table 2). There was no difference in posttreatment RI among three alpha-adrenergic antagonists. Posttreatment RI did not correlate with age, posttreatment PV, IPSS, BOOI or

\section{PdetQmax.}

We additionally explored the correlation between persistent urgency and reduction of RI after dutasteride treatment. In 20 patients (67\%) with persistent urgency after dutasteride, the reduction of RI was significantly less than in other 10 patients (33\%) without urgency after dutasteride (0.045 \pm 0.091 versus $0.096 \pm 0.042, p=0.04)$ (Table 3). Posttreatment PdetQmax and BOOI in patients with persistent urgency were significantly higher than in those without 
urgency after dutasteride (PdetQmax: $62 \pm 26 \mathrm{cmH}_{2} \mathrm{O}$ versus $40 \pm 17 \mathrm{cmH}_{2} \mathrm{O}, p=0.009$, BOOI: $46 \pm 28$ versus $24 \pm 20, p=0.019$ ), although these two parameters before dutasteride were not significantly different between the patients with and without persistent urgency (Table 3). Age and PV before and after dutasteride were not significantly different between the patients with and without persistent urgency. Total IPSS before and after dutasteride was significantly higher in patients with persistent urgency than in those without urgency after dutasteride. In patients with persistent urgency, prevalence of DO after dutasteride was 70\% (14 of 20 patients) compared to $40 \%$ (4 of 10 patients) in those without urgency after dutasteride $(\mathrm{p}=0.11)$. Prevalence of hypertension, hyperlipidemia and diabetes was not significantly different between the patients with and without persistent urgency.

\section{DISCUSSION}

The present study that was conducted with the same approach as our previous report ${ }^{4}$ suggests that bladder vascular resistance in patients with BPE is elevated in correlation with PV, and that whether urgency resolves or persists after dutasteride treatment is related to the degree of reduction of bladder vascular resistance along with the improvement of obstruction. These findings imply that reduction of obstruction and amelioration of bladder ischemia by dutasteride is an important factor for improvement of OAB symptoms in patients with LUTS associated with BPE.

In accordance with previous reports ${ }^{1,2}$, the present study confirmed that 24-week treatment of dutasteride reduced prostate size and improved voiding and storage symptoms including urinary urgency. We previously examined urodynamic effects of dutasteride on BOO and bladder storage function and showed that dutasteride relieved obstruction and improved bladder 
storage function. ${ }^{5}$ The present study confirmed the beneficial effect of dutasteride in relieving obstruction and improving bladder storage function. The present study further examined RI as a reflection of bladder vascular resistance before and after dutasteride treatment.

Overall RI significantly decreased after dutasteride treatment (from $0.548 \pm 0.069$ to 0.486 $\pm 0.064, p<0.001$ ), implying that dutasteride significantly increased bladder blood flow. It is well known that BOO induces bladder ischemia in men with LUTS/BPO. ${ }^{4,7,8}$ Plenty of animal experiments demonstrate the relationship between $\mathrm{BOO}$ and bladder ischemia. ${ }^{9-11}$ High voiding pressure, bladder overdistension and thick bladder wall associated with BOO seem to result in compression of intramural vessels and produce bladder ischemia. On the other hand, a previous study showed that thick bladder wall and bladder hypertrophy were reversibly improved after surgical relief of obstruction in men with BPE. ${ }^{12}$ Thus, relief of obstruction can lead to improvement of bladder blood flow through the reduction of intramural tension and bladder vascular resistance. Interestingly, the patients with no posttreatment urgency had lower BOOI and PdetQmax and better improvement of RI after dutasteride than those with persistent urgency. It seems that reduction of obstruction and improvement of bladder ischemia by dutasteride is a critical factor for resolution of OAB symptoms in patients with LUTS associated with BPE. If we compare pretreatment parameters between patients with or without persistent urgency after dutasteride (Table 3), those with persistent urgency had larger PV, higher IPSS, BOOI and PdetQmax than those without persistent urgency with a statistical significance noted only in IPSS. Because the number of patients was very small in the present study, further study with a large number of patients could identify pretreatment predictive factors for resolution of $\mathrm{OAB}$ symptoms with dutasteride treatment.

Our previous study showed that male OAB patients with low serum testosterone level at 
baseline had better improvement of OAB symptoms after dutasteride add-on therapy than those with normal testosterone level at baseline. ${ }^{13}$ The change of serum testosterone is considered as another factor accounting for improvement of bladder blood flow by dutasteride. Dutasteride is known to reduce serum and intraprostatic dihydrotestosterone concentrations by inhibition of 5 $\alpha$-reductase type 1 and $2 .{ }^{14,15}$ As a result, dutasteride causes a slight increase in serum testosterone level. The increase in serum testosterone after dutasteride treatment is higher in patients with low serum testosterone at baseline. ${ }^{16}$ Furthermore, a favorable level of serum testosterone was reported to have a protective effect on arterial aging, stiffness and elasticity. ${ }^{17}$ Therefore, increase in testosterone level by dutasteride might be a contributing factor to the improvement of bladder ischemia.

Despite a small number of the study patients, we believe that improvement of bladder blood flow by dutasteride is an important factor for resolution of OAB symptoms. During this study, Levovist ${ }^{\circledR}$, ultrasound contrast agent, has been discontinued and we could not increase further study patients. Further research with ultrasonography targeting bladder ischemia will lead to medical care with better quality for male patients with OAB associated with LUTS/BPO.

In conclusion, relief of obstruction and improvement of bladder ischemia might contribute to a beneficial impact of dutasteride on bladder storage function and storage symptoms.

\section{Disclosure}

There is no involvement, financial or otherwise. 


\section{REFERENCES}

1 Becher E, Roehrborn CG, Siami P et al: The effects of dutasteride, tamsulosin, and the combination on storage and voiding in men with benign prostatic hyperplasia and prostatic enlargement: 2-year results from Combination of Avodart and Tamsulosin Study. Prostate Cancer Prostatic Dis 2009; 12: 369-74.

2 Tsukamoto T, Shirai T, Sakamoto S et al: The effect of dutasteride on voiding and storage symptoms in men with benign prostatic hyperplasia. Acta Urol Jpn 2010; 56: 677-82.

3 Abrams P, Cardozo L, Fall M et al The standardization of the terminology of lower urinary tract function: Report from the standardisation sub-committee of the International Continence Society. Neurourol Urodyn 2002; 21: 167-78.

4 Wada N, Watanabe M, Kita M et al. Analysis of bladder vascular resistance before and after prostatic surgery in patients with lower urinary tract symptoms suggestive of benign prostatic obstruction. Neurourol Urodyn 2012; 31: 659-63.

5 Wada N, Kita M, Hashizume K et al. Urodynamic effects of dutasteride add-on therapy to alpha-adrenergic antagonist for patients with benign prostatic enlargement: prospective pressure-flow study. Neurourol Urodyn 2013; 32: 1123-7.

6 Abrams P. Bladder outlet obstruction index, bladder contractility index and bladder voiding efficacy: three simple indices to define bladder voiding function. BJU Int 1999; 84: $14-5$

7 Lin AT, Chen MT, Yang CH et al. Blood flow of the urinary bladder: effects of outlet obstruction and correlation with bioenergetic metabolism. Neurourol Urodyn 1995; 14: 285-92.

8 Mitterberger M, Pallwein L, Gradl J et al. Persistent detrusor overactivity after transurethral resection of the prostate is associated with reduced perfusion of the urinary bladder. BJU Int 
2007; 99: 831-35.

9 Matsumoto S, Chichester P, Kogan BA et al. The structural and vascular response of normal and obstructed rabbit whole bladders to distention. Urology 2003; 62: 1129-33.

10 Chichester P, Schroder A, Horan P et al. Vascular response of the rabbit bladder to chronic partial outlet obstruction. Mol Cell Biochem 2001; 226: 1-8.

11 Schroder A, Chichester P, Kogan BA et al. Effect of chronic bladder outlet obstruction on blood flow of the rabbit bladder. J Urol 2001; 165: 640-46.

12 Kojima M, Inui E, Ochiai A et al. Reversible change of bladder hypertrophy due to benign prostatic hyperplasia after surgical relief of obstruction. J Urol 1997; 158: 89-93.

13 Wada N, Hashidume K, Tamaki G et al. Add-on effect of dutasteride in patients with benign prostatic hyperplasia treated with alpha blocker : its effect on overactive bladder. Acta Urol Jap 2012; 58: 475-80.

14 Clark RV, Hermann DJ, Cunningham GR, et al. Marked suppression of dihydrotestosterone in men with benign prostatic hyperplasia by dutasteride, a dual 5 $\alpha$-reductase inhibitor. J Clin Endocrinol Metab 2004; 89: 2179-84.

15 Wurzel R, Ray P, Major-Walker K, et al. The effect of dutasteride on intraprostatic dihydrotestosterone concentrations in men with benign prostatic hyperplasia. Prostate Cancer Prostatic Dis 2007; 10: 149-54.

16 Hong SK, Min GE, Ha SB et al. Effect of the dual 5alpha-reductase inhibitor, dutasteride, on serum testosterone and body mass index in men with benign prostatic hyperplasia. BJU Int 2010; 105: 970-4.

17 Dockery F, Bulpitt CJ, Donaldson $\mathrm{M}$ et al. The relationship between androgens and arterial stiffness in older men. J Am Geriatr Soc 2003; 51: 1627-32. 


\section{Figure legend}

Figure 1. Transabdominal enhanced color Doppler ultrasonography 
Table1 Characteristics of study patients

\begin{tabular}{|c|c|}
\hline Age, yrs (range) & $73.6(60-83)$ \\
\hline Prostate volume, ml (range) & $68(30-139)$ \\
\hline \multicolumn{2}{|l|}{ 【Vascular risk factor】 } \\
\hline Hypertension, $n(\%)$ & $14(47 \%)$ \\
\hline Hyperlipidemia , $n(\%)$ & $6(20 \%)$ \\
\hline Diabetes , $n(\%)$ & $5(17 \%)$ \\
\hline IPSS, (range) & $18.8\left(4^{-} 32\right)$ \\
\hline QoL index, (range) & $4.6(2-6)$ \\
\hline \multicolumn{2}{|c|}{ 【Urodynamics parameters】 } \\
\hline Maximum flow rate, $\mathrm{ml} / \mathrm{s}$ (range) & $12.2\left(4.6^{-} 32.0\right)$ \\
\hline Postvoid residual, ml (range) & $93(0-455)$ \\
\hline BOOI, (range) & $58(7-134)$ \\
\hline PdetQmax, cmH2O (range) & $73(21-147)$ \\
\hline Detrusor overactivity & $23(77 \%)$ \\
\hline
\end{tabular}

BOOI: bladder outlet obstruction index 
Table2 Change of parameters before and after dutasteride treatment

\begin{tabular}{|c|c|c|c|}
\hline & Before dutasteride & 24 weeks after dutasteride & $P$ value \\
\hline IPSS & $18.8 \pm 7.7$ & $13.4 \pm 7.2$ & $<0.001$ \\
\hline Q1 (feeling of incomplete emptying) & $2.5 \pm 1.9$ & $1.7 \pm 1.7$ & 0.01 \\
\hline Q2 (increased daytime frequency) & $3.3 \pm 1.7$ & $2.3 \pm 1.7$ & $<0.001$ \\
\hline Q3 (intermittent stream) & $2.3 \pm 2.0$ & $1.7 \pm 1.6$ & $<0.05$ \\
\hline Q4 (urinary urgency) & $2.3 \pm 1.9$ & $1.4 \pm 1.4$ & $<0.01$ \\
\hline Q5 (slow stream) & $3.3 \pm 1.6$ & $2.4 \pm 1.5$ & $<0.01$ \\
\hline Q6 (straining) & $2.1 \pm 1.9$ & $1.6 \pm 1.5$ & 0.10 \\
\hline Q7 (nocturia) & $2.9 \pm 1.3$ & $2.3 \pm 1.2$ & $<0.001$ \\
\hline QoL index & $4.6 \pm 1.0$ & $3.4 \pm 1.3$ & $<0.001$ \\
\hline PV (ml) & $68 \pm 29$ & $48 \pm 28$ & $<0.001$ \\
\hline Serum PSA (ng/ml) & $6.9 \pm 11.1$ & $3.9 \pm 6.6$ & $<0.001$ \\
\hline Serum testosterone (ng/dl) & $\begin{array}{l}380 \pm 182 \\
\text { e uroflowmetry }\end{array}$ & $488 \pm 193$ & $<0.001$ \\
\hline Voided volume $(\mathrm{ml})$ & $192 \pm 107$ & $227 \pm 126$ & 0.07 \\
\hline $\mathrm{Qmax}(\mathrm{ml} / \mathrm{sec})$ & $12.2 \pm 6.1$ & $13.3 \pm 7.7$ & 0.09 \\
\hline \multicolumn{4}{|c|}{ 【Filling cystometry and PFS】 } \\
\hline Prevalence of DO, $n(\%)$ & $23(77)$ & $18(60)$ & $0.17^{*}$ \\
\hline PdetQmax (cmH2O) & $73 \pm 34$ & $54 \pm 25$ & $<0.001$ \\
\hline BOOI & $58 \pm 36$ & $38 \pm 27$ & $<0.001$ \\
\hline Bladder RI & $0.548 \pm 0.069$ & $0.486 \pm 0.064$ & $<0.001$ \\
\hline mean $\pm \mathrm{SD}$ & & & \\
\hline Using the Wilcoxon matched-pairs signet-ra & st except for pre & ce of DO & test* \\
\hline
\end{tabular}


Table3 Differences of each parameter between patients with and without urgency after dutasteride

\begin{tabular}{|c|c|c|c|c|}
\hline & & Persistent urgency & No persistent urgency & Pvalue \\
\hline No. patients, $n(\%)$ & & $20(67 \%)$ & $10(33 \%)$ & \\
\hline Reduction of bladder RI & & $0.045 \pm 0.091$ & $0.096 \pm 0.042$ & $<0.05$ \\
\hline Age (yrs) & & $73.8 \pm 5.6$ & $73.2 \pm 7.3$ & 0.84 \\
\hline \multirow[t]{2}{*}{ PV } & before dutasteride $(\mathrm{ml})$ & $71 \pm 28$ & $61 \pm 30$ & 0.37 \\
\hline & after dutasteride $(\mathrm{ml})$ & $51 \pm 28$ & $42 \pm 28$ & 0.44 \\
\hline \multirow[t]{2}{*}{ IPSS } & before dutasteride & $20.9 \pm 7.1$ & $14.6 \pm 7.4$ & $<0.05$ \\
\hline & after dutasteride & $15.8 \pm 7.0$ & $8.8 \pm 5.6$ & $<0.01$ \\
\hline \multirow[t]{2}{*}{ BOOI } & before dutasteride & $62 \pm 39$ & $49 \pm 30$ & 0.31 \\
\hline & after dutasteride & $46 \pm 28$ & $24 \pm 20$ & $<0.05$ \\
\hline \multirow[t]{2}{*}{ PdetQmax } & before dutasteride (cmH2O) & $78 \pm 35$ & $62 \pm 30$ & 0.20 \\
\hline & after dutasteride (cmH2O) & $62 \pm 26$ & $40 \pm 17$ & $<0.01$ \\
\hline \multirow[t]{2}{*}{ Prevalence of DO } & before dutasteride, $n(\%)$ & $16(80)$ & $7(70)$ & 0.52 \\
\hline & after dutasteride, $n(\%)$ & $14(70)$ & $4(40)$ & 0.11 \\
\hline \multirow[t]{3}{*}{ Vascular risk factors } & Hypertention , $n(\%)$ & $11(55)$ & $3(30)$ & 0.19 \\
\hline & Hyperlipidemia , $n$ (\%) & $5(25)$ & $1(10)$ & 0.33 \\
\hline & Diabetes , $n(\%)$ & $3(15)$ & $2(20)$ & 0.76 \\
\hline
\end{tabular}

mean \pm SD

Using the Wilcoxon matched-pairs signet-ranks test except for prevalence of DO and vascular risk factors using Chi-square test 


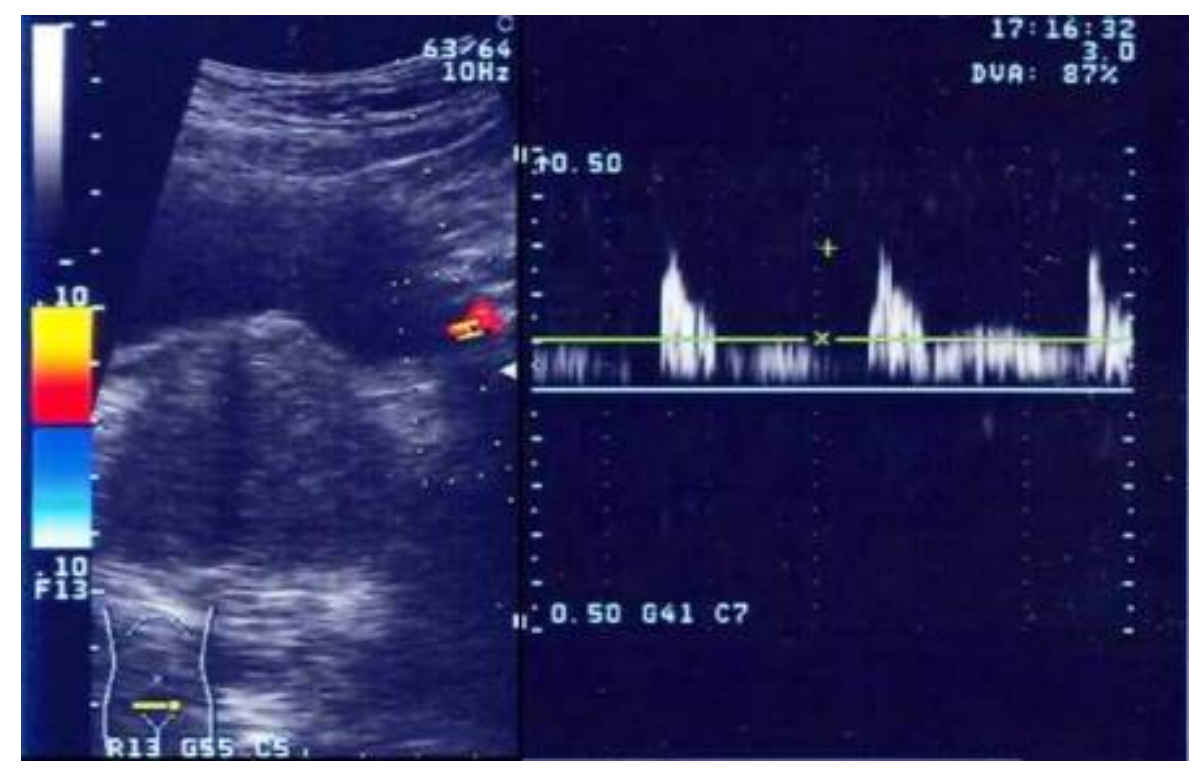

